\title{
CRISPR-mediated genome editing of Plasmodium falciparum malaria parasites
}

\author{
Marcus CS Lee ${ }^{1}$ and David A Fidock ${ }^{1,2^{*}}$
}

\begin{abstract}
The development of the CRISPR-Cas system is revolutionizing genome editing in a variety of organisms. The system has now been used to manipulate the genome of Plasmodium falciparum, the most lethal malaria-causing species. The ability to generate gene deletions or nucleotide substitutions rapidly and economically promises to accelerate the analysis of novel drug targets and to help elucidate the function of specific genes or gene families, while complementing genome-wide association studies.
\end{abstract}

\section{Genetic manipulation of the malaria parasite}

The global malaria burden exceeds 200 million infections per year, resulting in over 600,000 deaths annually. Most fatalities are attributable to blood-stage infections by the apicomplexan parasite Plasmodium falciparum. The current frontline treatments are artemisinin-based combination therapies, whose clinical use is at the scale of hundreds of millions of doses per year. The recent emergence of resistance to artemisinin in South East Asia is of global concern, given the devastating loss of the former frontline therapies chloroquine and pyrimethamine-sulfadoxine to drug-resistant strains of P. falciparum [1].

Genetic crosses and genome-wide association studies (GWAS) have been instrumental in uncovering candidate genes that are involved in resistance phenotypes. Nevertheless, confirmation of their actual contribution to resistance requires efficient tools to genetically modify $P$. falciparum parasites cultured in vitro. These transgenic lines could also be used to generate insights into the underlying mechanisms of drug action and parasite resistance. Genetic methodologies are also increasingly used to dissect molecular components of $P$. falciparum pathogenesis, including

\footnotetext{
* Correspondence: df2260@columbia.edu

'Department of Microbiology and Immunology, Columbia University College of Physicians and Surgeons, New York, NY 10032, USA

${ }^{2}$ Division of Infectious Diseases, Department of Medicine, Columbia

University College of Physicians and Surgeons, New York, NY 10032, USA
}

processes of host-cell invasion, protein trafficking inside the infected host cells, virulence, and immune evasion.

Steady progress has been made in transforming $P$. falciparum into a genetically tractable laboratory organism. Important milestones include the establishment of in vitro culture systems in 1979, transfection techniques in 1995, and the sequencing of the genome in 2002 [1]. Nevertheless, methods to engineer the $P$. falciparum genome have been hampered by limited tools and inefficiencies in transfection and integration. Furthermore, RNA interference (RNAi) is not effective in P. falciparum, unlike mammalian systems.

Gene disruption in $P$. falciparum traditionally requires one to three months of continuous culture before one can observe a stochastic homologous recombination event that leads to the integration of an episomally maintained plasmid by either a single- or a double-crossover (Figure 1A). Similar recombination-dependent approaches are used for allelic exchange, for example, to validate potential drugresistance mutations [1]. The inherent inefficiency of this process has precluded any large-scale gene deletion campaigns, with few exceptions such as the heroic effort that was successful in disrupting 53 out of 83 targeted genes that had potential roles in protein export into the host red blood cells (RBCs) [2]. As described below, genome editing techniques based on the RNA-guided CRISPR (clustered regularly interspaced short palindromic repeats)Cas (CRISPR-associated proteins) system have now been reported for $P$. falciparum [3], providing a powerful new approach that can be used to interrogate the malarial parasite genome.

\section{Harnessing the power of the double-strand break: genome editing using site-specific nucleases}

A double-strand break (DSB) in the chromosome of a cell is a potentially catastrophic event, and as a consequence, organisms have developed mechanisms to deal with this contingency. One emergency measure is non-homologous end joining (NHEJ), which simply stitches together the broken DNA ends in a process that can introduce small 


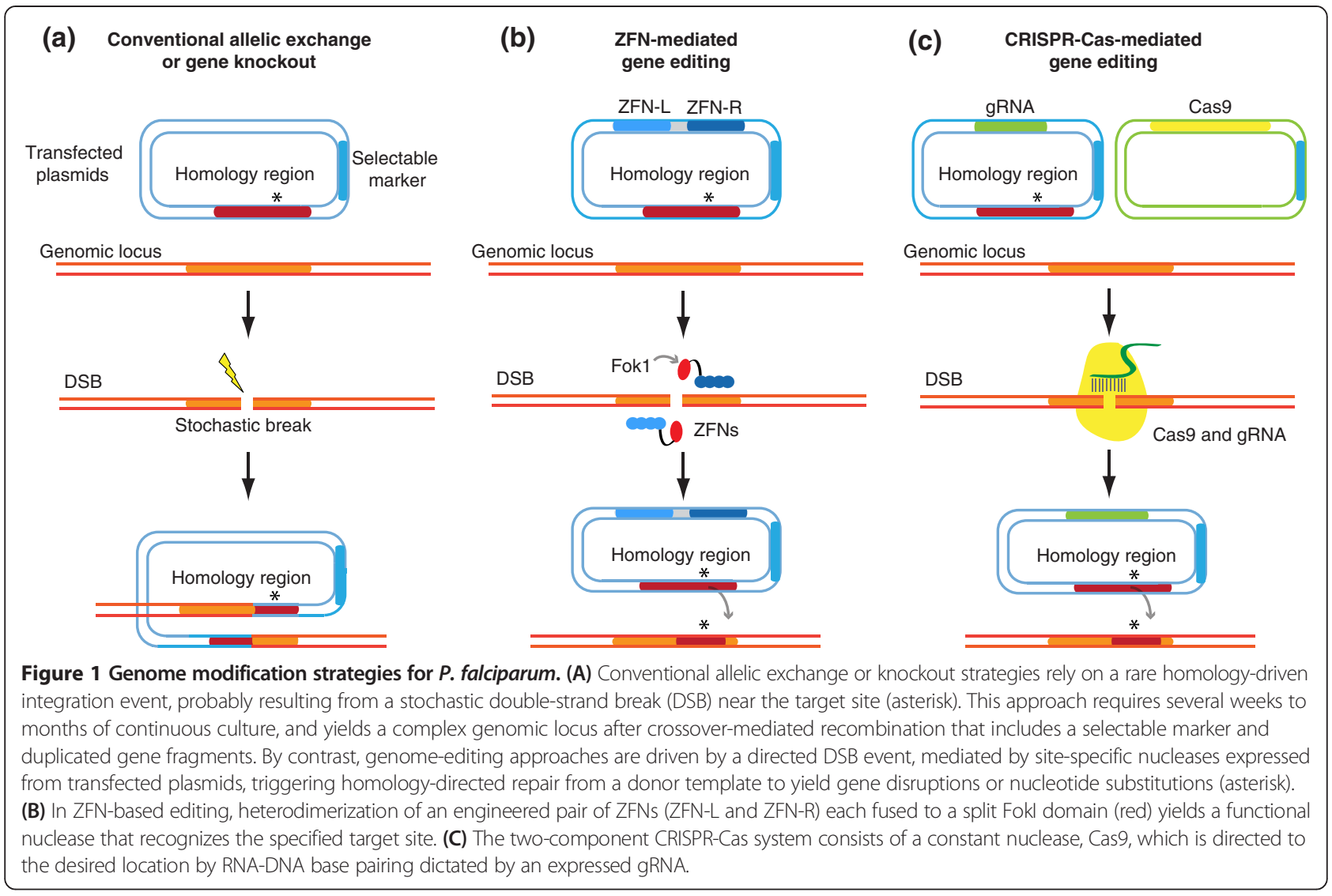

insertions or deletions. Alternatively, the cell can use the more precise method of homology-directed repair, using a homologous template to guide the repair [4]. Genome editing harnesses these processes experimentally to disrupt or modify genomic loci in defined ways. The common theme with all genome editing approaches is the use of a site-specific nuclease that cleaves the genome at a defined site, thus triggering a repair event that can be guided to yield outcomes ranging from complete gene deletion to more subtle sequence alterations [5].

Customized zinc-finger nucleases (ZFNs), in which the catalytic domain of the Fok1 nuclease is combined with multiple zinc-finger proteins that specify the DNA-binding site, were the first nucleases to be used widely for genome editing in a variety of organisms, including P. falciparum. These nucleases allowed parasites with targeted deletions or allelic replacements to be generated considerably faster than had been possible using conventional methods because ZFN-generated DSB events occur shortly after transfection, rather than weeks to months later [6]. More significantly, perhaps, template-guided repair of the DSB allows for the delivery of as little as a single-base change, an application ideally suited for testing the impact of putative drug resistance mutations (Figure 1B). The recombinant locus that results from ZFN editing contrasts with the complex locus formed by conventional allelic exchange, which often includes the selectable marker, thus precluding its use in downstream applications and a partial duplication of the targeted gene (resulting from crossovermediated plasmid integration; Figure 1A). ZFN editing is a powerful approach, but the cost and non-trivial nature of designing zinc-finger proteins that bind specific sequences necessitates specialized knowledge and limits the scale at which they can be deployed.

\section{A new platform for gene editing: CRISPR-Cas}

An alternative system for genome editing avoids the ZFN design problem by using RNA-DNA base pairing to guide DNA cleavage. CRISPR-Cas systems function in bacterial adaptive immunity as a defense against viruses or other invasive DNA. The CRISPR sequences, acquired initially from foreign DNA, are arrayed in the host bacterial genome and are transcribed and processed to generate short guide RNAs (gRNAs) that direct the Cas endonuclease to complementary target sites. The ease with which the Cas nuclease can be reprogrammed by supplying alternative gRNAs has led to its exceptionally rapid adoption as a genetic tool in a wide variety of organisms [7].

The first 20 nucleotides at the $5^{\prime}$ end of the gRNA determine specificity by homology to the target site, with an additional sequence requirement in the genome of an 
-NGG- 'protospacer adjacent motif' (PAM) immediately downstream of the target site. To date, the most common use in mammalian systems has been to generate loss-of-function alleles by directed cleavage followed by error-prone NHEJ. This approach has permitted not only the targeting of individual genes but also large-scale genetic screens that employ libraries of over 87,000 distinct gRNAs [8].

\section{CRISPR-Cas gene editing in Plasmodium parasites}

A new study by Ghorbal et al. [3] published in Nature Biotechnology has brought the power of the CRISPR-Cas system to $P$. falciparum. Two distinct applications, gene disruption and single-nucleotide gene editing, were demonstrated, highlighting the versatility of this approach. Parasites with disruptions at two non-essential loci, an integrated green fluorescent protein (GFP) reporter gene and the kahrp gene, which encodes a protein exported into the host $\mathrm{RBC}$, were efficiently obtained by insertion of a selectable marker within their coding sequences. Similarly effective were more subtle experiments that modified the sequences of two genes, orc1 and kelch13, which have putative roles in gene silencing and emerging resistance to artemisinin, respectively. Parasites that were altered at the appropriate sites were recovered with very high efficiency, despite there being no direct selection for the modification but only for the transfected plasmids, suggesting that neutral or even deleterious mutations can be generated using this system. As with earlier ZFN-editing experiments [6], the desired alterations were probably enriched within the bulk parasite population by the inclusion of silent mutations in the donor sequence that protected both the donor plasmid and edited genomic locus from cleavage. Furthermore, for reasons explained below, the ability to place the DSB site in very close proximity to the desired modification is another driver of efficient editing.

A two-plasmid approach was used by Ghorbal et al. [3] to express both the Cas9 nuclease and the gRNA (Figure 1C). Because of the requirement for a precise transcriptional start site for the gRNA, expression in mammalian cells has typically utilized the promoter of the U6 small nuclear RNA (snRNA) component of the spliceosome. U6 snRNA transcription is dependent on RNA polymerase III, which precisely transcribes a variety of non-protein-coding RNAs. Transcription of the U6 product initiates with a guanosine nucleotide, placing some constraint on the selection of target sequences in the genome, which optimally consist of the motif G- $\mathrm{N}_{19}$-NGG, with the final three nucleotides (the PAM sequence) critical for cleavage. One notable finding of the Ghorbal et al. study [3] is that the use of the P. falciparum U6 snRNA upstream regulatory region was able to drive expression of gRNAs in the parasite, but apparently without the functional requirement for the initial guanosine nucleotide. This is significant because it greatly expands the potential target sites within the parasite genome to any sequence with the -NGG PAM motif, a particularly liberating feature for an organism that has an average GC content of only $19.4 \%$.

One quirk of Plasmodium biology is that these parasites appear to lack the machinery for NHEJ [6]. Thus, the most basic and scaleable application of the CRISPR-Cas system, cleavage followed by error-prone repair to generate frameshift mutations, is not available to malaria researchers. Nevertheless, an enticing array of other possibilities awaits the field. The ability to express two or more gRNAs within the same cell may permit multiplex cleavage events that can generate large deletions, which could be used to investigate genome duplications that are often associated with drug resistance. In mammalian systems, a nuclease-dead version of Cas9, guided to a target gene, has been shown to act as a roadblock to transcription. Conversely, fusion of this inactive Cas9 to a transcriptional activation domain can increase gene expression [9]. Development of these gene regulatory approaches for Plasmodium would more than fill the gap left by the absence of RNAi tools for the parasite.

Robust, inexpensive methods to manipulate the parasite genome efficiently should now accelerate the discovery of new drug targets, the validation of GWAS data from field and clinical samples, and the identification of genes that are important for the critical parasite transitions between the human and mosquito hosts. The best new technologies offer not just improvements to existing methods but transform the very nature of the questions we can ask. The malaria parasite has joined the CRISPR-Cas revolution, and as these tools are brought to bear on a pathogen that is both deadly and biologically fascinating, we eagerly await the insights that are sure to follow.

\section{Note added in proof}

A recent report by Zhang et al. [10] describes the use of CRISPR/Cas systems to modify the genome of the rodent malaria parasite, Plasmodium yoelii.

\section{Abbreviations \\ Cas: CRISPR-associated proteins; CRISPR: clustered regularly interspaced short palindromic repeats; DSB: double-strand break; GFP: green fluorescent protein; gRNA: guide RNA; GWAS: genome-wide association studies; NHEJ: non-homologous end joining; PAM: protospacer adjacent motif; RBC: red blood cell; RNAi: RNA interference; snRNA: small nuclear RNA; ZFN: zinc-finger nuclease.}

\section{Competing interests}

The authors declare that they have no competing interests.

\section{Acknowledgements}

We thank Elizabeth Miller and Andrew Lee for critical reading of the manuscript. DAF gratefully acknowledges support from the US National Institutes of Health (Al50234 and Al109023). 


\section{Published online: 26 August 2014}

\section{References}

1. Anderson T, Nkhoma S, Ecker A, Fidock D: How can we identify parasite genes that underlie antimalarial drug resistance? Pharmacogenomics 2011, 12:59-85.

2. Maier AG, Rug M, O'Neill MT, Brown M, Chakravorty S, Szestak T, Chesson J, Wu Y, Hughes K, Coppel RL, Newbold C, Beeson JG, Craig A, Crabb BS, Cowman AF: Exported proteins required for virulence and rigidity of Plasmodium falciparum-infected human erythrocytes. Cell 2008, 134:48-61.

3. Ghorbal M, Gorman M, Macpherson CR, Martins RM, Scherf A, Lopez-Rubio JJ: Genome editing in the human malaria parasite Plasmodium falciparum using the CRISPR-Cas9 system. Nat Biotechnol 2014, doi: 10.1038/nbt.2925.

4. Lee AH, Symington LS, Fidock D: DNA repair mechanisms and their biological roles in the malaria parasite Plasmodium falciparum. Microbiol $\mathrm{Mol}$ Biol Rev in press.

5. Urnov FD, Rebar EJ, Holmes MC, Zhang HS, Gregory PD: Genome editing with engineered zinc finger nucleases. Nat Rev Genet 2010, 11:636-646.

6. Straimer J, Lee MC, Lee AH, Zeitler B, Williams AE, Pearl JR, Zhang L, Rebar EJ, Gregory PD, Llinás M, Urnov FD, Fidock DA: Site-specific genome editing in Plasmodium falciparum using engineered zinc-finger nucleases. Nat Methods 2012, 9:993-998.

7. Sander JD, Joung JK: CRISPR-Cas systems for editing, regulating and targeting genomes. Nat Biotechnol 2014, 32:347-355.

8. Koike-Yusa H, Li Y, Tan EP, Velasco-Herrera Mdel C, Yusa K: Genome-wide recessive genetic screening in mammalian cells with a lentiviral CRISPR-guide RNA library. Nat Biotechnol 2014, 32:267-273.

9. Gilbert LA, Larson MH, Morsut L, Liu Z, Brar GA, Torres SE, Stern-Ginossar N, Brandman O, Whitehead EH, Doudna JA, Lim WA, Weissman JS, Qi LS: CRISPR-mediated modular RNA-guided regulation of transcription in eukaryotes. Cell 2013, 154:442-451.

10. Zhang C, Xiao B, Jiang Y, Zhao Y, Li Z, Gao H, Ling Y, Wei J, Li S, Lu M, Su XZ, Cui H, Yuan J: Efficient Editing of Malaria Parasite Genome Using the CRISPR/Cas9 System. MBio 2014, 5 .

doi:10.1186/s13073-014-0063-9

Cite this article as: Lee and Fidock: CRISPR-mediated genome editing of Plasmodium falciparum malaria parasites. Genome Medicine 2014 6:63. 